\title{
Multi-population Ensemble Particle Swarm Optimizer based Energy Efficient Clustering Algorithms for IOT Applications
}

\author{
Roshnee Adlak \\ M.Tech Scholar \\ Department of Computer Science and \\ Engineering \\ Radharaman Institute of Technology \\ $\&$ Science \\ Bhopal, Madhya Pradesh, India
}

\author{
Pooja Meena \\ Assistant Professor \\ Department of Computer Science and \\ Engineering \\ Radharaman Institute of Technology \& \\ Science \\ Bhopal, Madhya Pradesh, India
}

\author{
Chetan Agrawal \\ Assistant Professor
}

Department of Computer Science and

Engineering

Radharaman Institute of Technology \&

Science

Bhopal, Madhya Pradesh, India

\begin{abstract}
Now-a-days with growing technology such as wireless sensor networks (WSN) are developed with advancement of Internet-of-Things (IoT). While deploying these applications practically, there may arise associated issues. Among all available issues, the major concern is energy utilization while data communication among these resource limited sensors. In this paper we have proposed a cluster-based routing protocol for IoT with benefits of machine learning to predict the energy wastage. Further, we proposed multi-population ensemble particle swarm optimizer (MPEPSO) for cluster head selection and cluster updation. The simulation was performed on MATLAB platform and shows its efficacy over other algorithms.

Keywords: Internet of Things (IoT), Wireless Sensor Network, Optimization, Energy Efficiency.
\end{abstract}

\section{INTRODUCTION}

The Internet of Things (IoT) has recently emerged with new applications and services to connect and give access to all kinds of devices from the physical world. Wireless sensor networks (WSNs) are one of the key infrastructures that provide support to the IoT paradigm. They consist of a set of small electronic devices, commonly called nodes, which share and collect information about their surroundings. The success of wireless sensor network in the form of technology and applications in different areas like home automation, industrial applications, security and military surveillance, and many more raises the further need for machine-to-machine connectivity and availability of the data or information anytime and anywhere [1]. This requirement leads to the new technology development in the form of Internet of Things (IoT). The IoT allows the connectivity among the devices and helps in acquisition of data or information at any time and from any place. There are numerous applications of IoT coming up using different technologies [2]. Also, IoT enables innovative services in numerous applications like smart transportation, smart home, smart city, smart lifestyle, smart retail, smart agriculture, smart industries, smart emergency, smart health care, smart environment, and many more [3, 4]. The use of these applications and their demand has increased the scope of research and innovation in this domain $[2,5]$.

The most significant part of above-mentioned applications using IoT needs sensing and monitoring the environment or acquiring the data from different IP-enabled devices or sensors. The sensor devices used for sensing and monitoring are battery-operated and energy-constrained. This implies that power consumption and energy are critical aspects. IP-based communication effectively utilizes more energy, which leads these low-powered devices to deplete rapidly. These huge numbers of devices communicate and collaborate with each other in order to accomplish a given job or task. This raises the need for effective connectivity and efficient communication among these devices in an optimized way, which is a very challenging task. In this context, there is a need for a solution that promises maximal connectivity through minimum communication. The most efficient way to fulfill these needs is to collaborate among the devices or sensors and perform the tasks for a given application. One way to achieve this is through grouping the devices in an efficient way in terms of energy usage and computational complexity.

For any IoT-based application, it is required to collect the data through sensing devices and process these data through different algorithms. Then, the processed information can be accessed through the Internet anywhere and at any time. The grouping of devices or sensors is known as clustering. Clustering helps in carrying out the task of acquiring the information in an efficient way with minimum number of communications within a network [6] and disseminating this information for further processing. Clustering also helps in prolonging the network lifetime and further the lifetime of an IoT-based application that is deployed for a specific task.

To achieve this, we introduce two-layer architectural framework consisting of two layers: upper layer that 
consists of IP-enabled IoT devices and lower layer that is simply sensor devices. In this regard, for grouping the devices, clustering algorithms are employed. In clustering, each cluster chooses one node within the cluster as a cluster head, and further the communication among the nodes in a network is carried out through the cluster head. If two nodes in a network are not within the range of each other, multihop communication is required. Communication through cluster head avoids multihop communication up to some extent. The cluster head also helps in aggregating the data acquired by different nodes in a network. In IoT-based network, as mentioned, two-layer architecture consists of sensor layer and IoT layer. The grouping of these devices is possible in two ways, that is, from sensor layer to IoT layer and vice versa.

Clustering offers numerous advantages. It supports network scalability]. Routing decisions within a cluster and handles by the respective cluster head thereby reducing the routing overhead. Clustering saves communication bandwidth as it checks the communication between Cluster heads $(\mathrm{CH})$, thus avoiding repetitive exchange of data packets among nodes [7]. By stabilizing the network at the cluster level, the overhead for topology maintenance is reduced drastically. The end devices only concern themselves with connecting to their $\mathrm{CHs}$ without being affected by changes at the inter- $\mathrm{CH}$ tier. Only the $\mathrm{CHs}$ and gateway nodes form the backbone network, resulting in much simpler topology, less overhead, flooding and collision. A CH aggregates data collected by sensors within its cluster which leads to reduction in the number of packets exchanged. By utilizing advanced management strategies for con-serving device battery, a $\mathrm{CH}$ extends the lifetime of network [7]. A CH can implement various scheduling algorithms to ensure that nodes operate in low-power mode when not active to conserve battery resources. For instance, nodes can be engaged in a round-robin fashion by specifying transmission and reception timeslots so that collisions are avoided and redundancy minimized.

\section{LITERATURE REVIEW}

According to the clustering protocol, cluster head is elected in each iteration or round which requires the formation of new clusters regularly. This may lead to excessive utilization of energy due to routing overhead, which may not be acceptable for any mobile devices, especially in IoT devices. So, there is requirement of an energy-efficient $\mathrm{CH}$ replacement method that can be employed in order to avoid utilization of extra energy in cluster formation and transmission of advertisement message to cluster members. The unequal clustering routing algorithm is needed to be focused and improved for IoT applications with dynamic number of nodes as well as mobile nodes. This will lead to reduction of network energy consumption and extends the network life cycle with higher stability. Some contribution of researchers are discussed below:

Ullah et al. [1] proposed a data aggregation scheme based on clustering of the nodes and extreme learning machine (ELM) which efficiently reduces redundant and erroneous data. Mahalanobis distance-based radial basis function is applied to the projection stage of the ELM to reduce the instability of the training process. Kalman filter is also used to filter the data at each sensor node before transmitted to the cluster head.

Radhika et al. [2] presented a modified clustering methodology that diminishes the overhead in clustering and message exchanges thereby effectively scheduling the clustering task. The network is clustered subject to the remaining energy of sensor nodes. Energy based parameters decide cluster head nodes and ancillary nodes and the member nodes are linked with them. The roles of the head nodes of the cluster are interchanged depending on the nodes' states. Reclustering of nodes is accomplished to achieve minimum energy consumption by calculating the update cycle using a fuzzy inference system.

Manzoor et al. [3] focused on improving the Two-Level Hierarchy for Low Energy Adaptive Clustering Hierarchy (TL-LEACH) protocol to provide energy efficiency; in terms of communication overhead and making the communication among the end-nodes, cluster-heads and base station as robust as possible. Two major drawbacks of the TL-LEACH protocol have been focused in this research that are mainly related to using the protocol for large-scale WSN and making the communication among the nodes robust. A novel cluster-head selection mechanism has been introduced to improve the energy-efficiency of the TLLEACH and the new version has been named as Extended TL-LEACH (ETL-LEACH).

Madhumathy et al. [4] proposed an agent cluster-based routing protocol which will subdivide the cluster into independent subgroups according to the satisfaction list. The independent sub groups are associated with an agent node that can communicate to the cluster head. The proposed agent cluster-based routing algorithm is created keeping into consideration to reduce the energy consumption while transmitting data from node to cluster head. The agent node is chosen based on the satisfaction list which reduces the chances of agent node die out problem thus enhancing the network reliability and lifetime.

Behera et al. [5] modified the existing low-energy adaptive clustering hierarchy (LEACH) clustering protocol is modified by introducing a threshold limit for cluster head selection with simultaneously switching the power level between the nodes. The proposed modified LEACH protocol outperforms as compared to the existing LEACH protocol with $67 \%$ rise in throughput and extending the number of alive nodes to 1750 rounds which can be used to enhance the WSN lifetime. When compared with other energy efficient protocols, it is found that the proposed algorithm performs better in terms of stability period and network lifetime in different scenarios of area, energy and node density.

Razzaq et al. [6] proposed K-means clustering-based routing protocol and considers an optimal fixed packet size according to the radio parameters and channel conditions of the transceiver. This approach can minimize the energy consumption of individual node and increase the network lifetime as a whole.

\section{METHODOLOGY}


Clustering has proved to be among the more potent strategies for enhancing system capacity and creating an energy-efficient WSN routing algorithm. Furthermore, as previously stated, model-based WSN management has significant disadvantages. The routing method is intended to adhere to different QoS requirements in order to improve efficiency and extend the lifetime of WSN challenges and issues evaluated by the network. As a result, this segment discusses several optimization techniques that assist to WSN energy efficiency and give implementation guarantee for Quality of Service (QoS).

\section{A. Network Diagram}

The design assumption used in this investigation is as follows:

$>\mathrm{N}$ sensors are typically distributed over the sensing zone, which is $A=N^{*} N$ in size. When both sensors and the base stations are randomly placed.

$>$ Each network device has an unique Registration identification as well as identical starting energies. The nodes have a finite quantity of energy, whereas the Base Station has an infinite supply.

$>$ The link is symmetrical. The node can determine the distance between the transmitter and itself based on the collected signal intensity.

$>$ Each node needs just one timeslot to link up with its parent node, and each node may only take or transmit single data packet and corresponding control packet within that period. The node's transmit power may be changed dependent on the contact range.

\section{B. Prototype of Energy Usage}

The quantity of energy used by sensor nodes is spent by exchanging data. In this study, we just examine the energy use price of information transmission and combining. The energy consumption of sending and receiving is codified in the following computations in equation (4.1):

$$
\begin{gathered}
E_{T X}(M, S) \\
= \begin{cases}M E_{\text {selects }}+M \varepsilon_{f s} S^{2}, & S<S_{0} \\
M E_{\text {selects }}+M \varepsilon_{a m p} S^{4} & S \geq S_{0}\end{cases} \\
\mathrm{E}_{\mathrm{RX}}(M)=M E_{\text {selects }}
\end{gathered}
$$

Here $\mathrm{M}$ is the length of the data.

$\mathrm{S}$ denotes the length or duration of data transfer.

$\mathrm{E}_{\text {selects }}=$ the amount of energy used through transmission and reception of unit length data.

$\varepsilon f s$ and $\varepsilon a m p=$ amplifier energy use in the free space model and the multiple path attenuation model, respectively.

Whenever the range $\mathrm{S}$ between the transmitting and reception nodes is less than the energy use model threshold $\mathrm{S} 0$, the free space model is used, and the transmit power is attenuated as S2. The multi-path attenuation architecture could be used instead, with S4 as the transmission power. The energy required by nodes to combine M-length data is determined as follows:

$$
E_{u}(M)=M E_{d a}
$$

Here Eda is the amount of energy needed to combine a unit quantity of data.

\section{A. Multi-population Ensemble Particle Swarm Optimizer} (MEPSO)

MEPSO's core idea is to combine PSO exploring methods with diverse characteristics into a single algorithm and flexibly assign particles to the best-performing exploring approach. As a result, the favoured PSO searching method might employ additional computing resources to boost the effectiveness of the suggested algorithm. MEPSO particles are categorized into three indicative sub-populations and one reward sub-population. Every indicative subpopulation does have the same tiny amount of particles, and particles in various indicator sub - populations modify their velocities in various manner. Particles in the three indicative sub - populations modify their velocities using the LDWPSO, UPSO, and CLPSO strategies, respectively, in this study. A learning period is specified as a set number of rounds. So at end of a learning period, the effectiveness of each indicative sub-population is assessed to determine the best-performing exploring approach, and then the reward sub-population is assigned to the associated strategic approach. In the suggested method, Figure 1 depicts a multi-population of twenty-five particles. Each indication subpopulation contains five particles, while the reward sub-population has ten particles. The reward subpopulation particles are instantaneously assigned to the best-performing indicative sub-population.

Proposed Algorithm MPEPSO

MPEPSO contains single reward sub-population $P O P_{r}$ and three indicator sub - populations represented by $P O P_{H}$, wherein $H \in 1,2,3$ is the PSO exploring technique indexing that correlates to the LDWPSO, UPSO, and CLPSO. The percentage of $P O P_{H}$ in the population is denoted by $h$. The number of particles in the indicative sub - populations is determined by the amount of $h$.

The indication sub-population $N_{H}$ population size is calculated using the following formula:

$$
N_{H}=\left[N * \lambda_{H}\right]
$$

Strategy $\mathrm{H}$ updates the velocities of the particles in $P O P_{H}$. Consequently, the quantity of particles in the reward subpopulation $\mathrm{Nr}$ may be calculated as follows:

$$
N_{r}=N-\sum_{H=1,2,3} N_{H}
$$

The strategy enhancement $\Delta F_{H}$ will be documented as :

$$
\Delta F_{H}=\Delta F_{H}+f\left(\text { pbest }_{i}\right)-f\left(x_{i}\right), \quad i \in P O P_{H} \quad \text { (vi) }
$$

The method that performs the best will be chosen and listed by $\mathrm{K}$ :

$$
K=\arg \left(_{H=1,2,3}^{\max }\left(\frac{\Delta F_{H}}{\left[N * N_{H}\right]}\right)\right.
$$

$P O P_{r}$ represents the reward population. Particles in $P O P_{r}$ will be assigned to $P O P_{K}$ and their velocities will be updated using the best-performing method $\mathrm{k}$. The suitable PSO exploring technique will have much more particles to discover a better alternative by flexibly assigning the reward subgroup to a good performing method at a particular learning period. As a result, MPEPSO's performance is projected to improve. Throughout the calculation process, the learning time is utilized to finetune the learning pace.

Multi-population Ensemble PSO




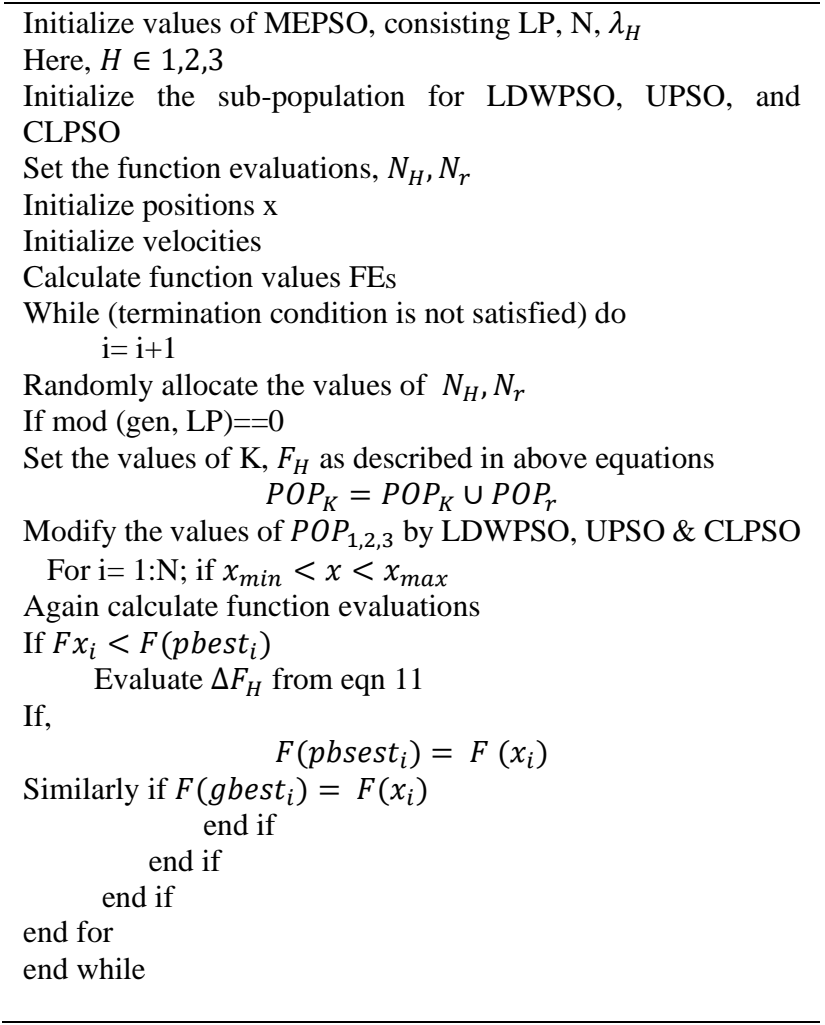

\section{RESULT ANALYSIS}

The simulation is performed on the MATLAB platform beneath various situations and with varied settings to evaluate the effectiveness of the suggested model. These variables are addressed more below.

Throughput: It is also an essential statistic that is determined regarding the successful transmission of packets of data to the sink node at a specific time. IoT routing methods are designed to enhance throughput.

Network Longevity: Because sensor nodes are batterypowered, network endurance is the most significant metric in IoT routing protocol. This is determined by counting the number of alive and dead nodes after each round sometimes after a set length of time.

Simulation Scenario is presented in table 1.

Table 1. Simulation scenario

\begin{tabular}{|l|l|}
\hline Simulation Scenario & Values \\
\hline Area & $100 \mathrm{~m}^{*} 100 \mathrm{~m}$ \\
\hline Sensor nodes & $50-100$ \\
\hline Initial energy of network & $50 \mathrm{~J}$ \\
\hline Energy Dissipation while transmitting bits & $16.7 \mathrm{~nJ} / \mathrm{bits}$ \\
\hline Energy Dissipation while receiving bits & $36.1 \mathrm{~nJ} / \mathrm{bits}$ \\
\hline $\begin{array}{l}\text { Energy Dissipation during amplification } \\
\text { of power }\end{array}$ & $1.98 \mathrm{~nJ} / \mathrm{bits}$ \\
\hline Packet size & $2000,3000,4000$ \\
\hline
\end{tabular}

Figure 1 represents the simulation result of number of packets transmitted to base station and similarly, figure 2 represents the simulation result for energy utilization by all nodes in the network. Table 2 represents the comparative result analysis of the proposed methodology with existing works.

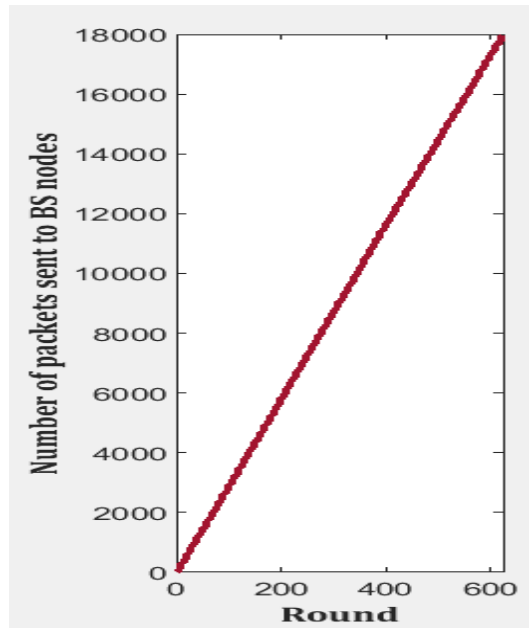

Fig.1. Number of Packets transmitted to Base Station

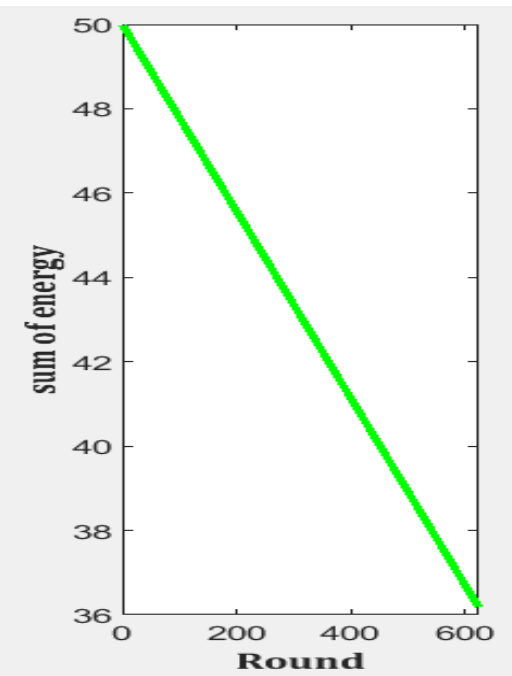

Fig.2. Energy Utilization of Network

Table 2. Comparative Performance Evaluation

\begin{tabular}{|l|l|l|l|l|}
\hline Parameters & Rounds & $\begin{array}{l}\text { MLEAC } \\
\text { H [5] }\end{array}$ & PSO [11] & Proposed \\
\hline $\begin{array}{l}\text { First Dead } \\
\text { Node }\end{array}$ & - & 1200 & 2100 & 3234 \\
\hline $\begin{array}{l}\text { Network } \\
\text { Longevity }\end{array}$ & - & $\begin{array}{l}7550 \\
\text { rounds }\end{array}$ & $\begin{array}{l}7600 \\
\text { rounds }\end{array}$ & $\begin{array}{l}>8000 \\
\text { rounds }\end{array}$ \\
\hline $\begin{array}{l}\text { Residual } \\
\text { Energy }\end{array}$ & $\begin{array}{l}2000 \\
\text { round }\end{array}$ & $2.24 \mathrm{~J}$ & $1.91 \mathrm{~J}$ & $10 \mathrm{~J}$ \\
\hline
\end{tabular}

\section{Conclusion}

While successful deployment of IoT, one of the major challenging factors in IoT and its application is energy efficiency of sensor nodes. To cope with this issue, the most important factor is to select the data forwarding node to sink node. In this paper, we have considered it as optimization problem. The paper also presented comparative result analysis on the parameters such as network longevity and residual energy. From result analysis it can be clearly concluded that the proposed 
algorithm based routing protocol outperforms better result as compared to existing works.

\section{References}

[1] Ullah, I.; Youn, H. Y. Efficient Data Aggregation with Node Clustering and Extreme Learning Machine for WSN. J. Supercomput. 2020, $76 \quad$ (12), 10009-10035. https://doi.org/10.1007/s11227-020-03236-8.

[2] Radhika, S.; Rangarajan, P. On Improving the Lifespan of Wireless Sensor Networks with Fuzzy Based Clustering and Machine Learning Based Data Reduction. Appl. Soft Comput. J. 2019, 83, 105610. https://doi.org/10.1016/j.asoc.2019.105610.

[3] Manzoor, K.; Jokhio, S. H.; Khanzada, T. J. S.; Jokhio, I. A. Enhanced TL-LEACH Routing Protocol for Large-Scale WSN Applications. In Proceedings - 2019 Cybersecurity and Cyberforensics Conference, CCC 2019; Institute of Electrical and Electronics Engineers Inc., 2019; pp 35-39. https://doi.org/10.1109/CCC.2019.00-12.

[4] Banerjee, I.; Madhumathy, P. An Agent Cluster Based Routing Protocol for Enhancing Lifetime of Wireless Sensor Network. In 1st International Conference on Advanced Technologies in Intelligent Control, Environment, Computing and Communication Engineering, ICATIECE 2019; Institute of Electrical and Electronics Engineers Inc., 2019; pp 265-268. https://doi.org/10.1109/ICATIECE45860.2019.9063788.

[5] Behera, T. M.; Samal, U. C.; Mohapatra, S. K. Energy-Efficient Modified LEACH Protocol for IoT Application. IET Wirel. Sens. Syst. 2018, 8 (5), 223-228. https://doi.org/10.1049/ietwss.2017.0099.

[6] Razzaq, M.; Devi Ningombam, D.; Shin, S. Energy Efficient KMeans Clustering-Based Routing Protocol for WSN Using Optimal Packet Size. In International Conference on Information Networking; IEEE Computer Society, 2018; Vol. 2018-January, pp 632-635. https://doi.org/10.1109/ICOIN.2018.8343195.

[7] Wibisono, W.; Ahmad, T.; Anggoro, R. Position-Based Scheme for Multi-Hop Routing Protocol in Cluster-Based Wireless Sensor Networks. In Proceeding of 2018 4th International Conference on Wireless and Telematics, ICWT 2018; Institute of Electrical and Electronics $\quad$ Engineers $\quad 2018$. https://doi.org/10.1109/ICWT.2018.8527735.

[8] Abidoye, A. P.; Obagbuwa, I. C. Models for Integrating Wireless Sensor Networks into the Internet of Things. IET Wirel. Sens. Syst. 2017, 7 (3), 65-72. https://doi.org/10.1049/iet-wss.2016.0049.

[9] Wu, W.; Xiong, N.; Wu, C. Improved Clustering Algorithm Based on Energy Consumption in Wireless Sensor Networks. IET Networks 2017, 6 (3), 1-7. https://doi.org/10.1049/ietnet.2016.0115.

[10] Li, P.; Jiang, W.; Xu, H.; Liu, W. Energy Optimization Algorithm of Wireless Sensor Networks Based on LEACH-B. In Lecture Notes on Data Engineering and Communications Technologies; Springer, 2017; Vol. 1, pp 391-404. https://doi.org/10.1007/978-3-31949109-7_37.

[11] N. Bilandi, H. K. Verma, and R. Dhir, "Performance and evaluation of energy optimization techniques for wireless body area networks," Beni-Suef Univ. J. Basic Appl. Sci. 2020 91, vol. 9, no. 1, pp. 1-11, Sep. 2020, doi: 10.1186/S43088-020-00064-W. 